\title{
Genotipo complejo en un paciente con GIST
}

\author{
Complex genotype in a GIST patient
}

\author{
Henry Becerra', Andrés Felipe Cardona $a^{1,2}$, Andrés Acevedo33, Carlos Vargas ${ }^{1,2}$, Hernán Carranza',2, Jorge Otero, ${ }^{1,2}$, \\ Johanna Álvarez ${ }^{4}$, Diego Aguirre ${ }^{5}$, Silvia Serrano², Alirio Zuluaga ${ }^{6}$ \\ 'Grupo Oncología Clínica y Traslacional, Instituto de Oncología, Fundación Santa Fe de Bogotá (Bogotá, Colombia). \\ 2 Fundación para la Investigación Clínica y Molecular Aplicada del Cáncer (FICMAC); investigador asociado ONCOLGroup. \\ ${ }^{3}$ Grupo Hematología y Trasplante de Médula Ósea, Instituto de Oncología, Fundación Santa Fe de Bogotá (Bogotá, Colombia). \\ ${ }^{4}$ Departamento de Laboratorio y Patología, Fundación Santa Fe de Bogotá (Bogotá, Colombia). \\ 5 Departamento de Imágenes Diagnósticas, Fundación Santa Fe de Bogotá (Bogotá, Colombia). \\ ${ }^{6}$ Grupo Hematooncología, Fundación Cardioinfantil (Bogotá, Colombia).
}

\begin{abstract}
Resumen
Se presenta el caso de un hombre de 35 años diagnosticado de un tumor del estroma gastrointestinal (GIST) localizado en el yeyuno proximal y estratificado como de alto riesgo, en quien se resecó el primario sin evidencia de recaída, hasta documentar múltiples lesiones hepáticas, por lo que inició imatinib $\left(\mathrm{Glivec}^{\circledR}\right) 400 \mathrm{mg} /$ día. Después de encontrar progresión, se aumentaron las dosis del inhibidor sin respuesta, por lo que inició sunitinib (Sutent $\left.{ }^{\circledR}\right) 50$ mg/día con el mismo resultado. Posteriormente, se realizó una reevaluación del histotipo y genotipificación, encontrando positividad para la mutación del exón 9 del gen KIT. A partir de noviembre del 2009, se administró sorafenib (Nexavar $\left.{ }^{\circledR}\right) 400$ mg cada 12 horas, logrando una respuesta metabólica superior al 50\% (evaluada por tomografía por emisión de positrones -PET/TAC-) y parcial siguiendo los criterios descritos por Choi y colaboradores. Durante el tratamiento de tercera línea, se efectuó una nueva biopsia del tumor, que demostró un genotipo complejo, incluyendo la mutación V600E del BRAF, y manteniendo el beneficio clínico con la intervención descrita hasta julio del 2011; en el momento, el paciente continúa vivo. A continuación, se plantea un análisis de los mecanismos moleculares que explican la resistencia a los inhibidores de tirosina-quinasa y las implicaciones sobre la terapéutica del GIST.
\end{abstract}

Palabras clave: GIST, BRAF, CKIT, patología molecular, sorafenib, resistencia.

\begin{abstract}
The case of a 35-year-old man is presented; he was diagnosed as having a gastrointestinal stromal tumour (GIST) which was located in the proximal jejunum and stratified as being high risk. The primary GIST was resected without evidence of relapse until multiple hepatic lesions were documented so that treatment with $400 \mathrm{mg} /$ day imatinib $\left(\mathrm{Glivec}^{\circledR}\right)$ was begun. After progression was found, the inhibitor dose was increased without response, meaning that $50 \mathrm{mg} /$ day sunitinib (Sutent ${ }^{\circledR}$ ) was begun with the same result. The histotype and genotyping were thus re-evaluated, positivity for KIT gene exon 9 mutation being found; 400 mg BID sorafenib (Nexavar ${ }^{\circledR}$ ) was administered from November 2009 onwards, achieving a 50\% metabolic response (evaluated by PET/CAT) and partial response following the criteria described by Choi and coworkers. A new biopsy of the tumour was made during third-line treatment, demonstrating a complex genotype including the BRAF V600E mutation. Clinical benefit was maintained with the aforementioned intervention until July 2011; the patient was still alive at this point. A detailed analysis of the molecular mechanisms explaining resistance to tyrosine kinase inhibitors and the implications concerning GIST therapy was then proposed.
\end{abstract}

Key words: GIST, BRAF, CKIT, molecular pathology, sorafenib, resistance.

\section{Introducción}

Los tumores del estroma gastrointestinal (GIST, por su sigla en inglés) se clasificaban anteriormente como leiomiomas, leiomiosarcomas o leiomioblastomas, y en la actualidad se consideran como una entidad nosológica y clínicamente diferente. El GIST representa el tumor mesenquimal más frecuente originado en el tracto gastrointestinal y comprende cerca del 3\% de estas neoplasias'. Se ha demostrado que se originan a partir de las células intersticiales de Ramón y Cajal, presentes en el intersticio de la muscularis propia del tracto gastrointestinal. Estos elementos formes hacen parte de los plexos mientéricos de la pared de las vísceras abdominales, y su función principal es la regulación de la motilidad intestinal mediante la generación y propagación de ondas de despolarización lenta, que se traducen en la formación efectiva del peristaltismo 2,3 . 
En orden de frecuencia, se han descrito en el estómago (41\%-45\%), intestino delgado (23\%-32\%) y otros sitios del tracto gastrointestinal ${ }^{4-6}$. La caracterización epidemiológica de la enfermedad en Colombia ha permitido determinar que la edad promedio de presentación se encuentra en la quinta década, sin ninguna dominancia de género. En nuestro entorno, suele diagnosticarse como enfermedad metastásica en el $45 \%$ de los individuos que se consideran candidatos para recibir tratamientos con inhibidores de tirosinaquinasa multiblanco ${ }^{5,6}$.

Históricamente el tratamiento de elección para los sujetos con lesiones resecables es la cirugía”; sin embargo, para quienes tienen una enfermedad irresecable o metastásica, el manejo se concentra en el control sistémico ${ }^{7,8}$. En Colombia, se ha descrito una mediana de supervivencia global (SG) para los pacientes con GIST de 46 meses (rango 4,2 a 54,2 meses), tiempo que resulta menor para aquellos que debutan con metástasis (31 meses) y para quienes tienen restricciones de acceso a la medicación ${ }^{5}$. A la fecha, el desarrollo de la biología molecular tumoral ha estimulado la búsqueda de nuevas alternativas terapéuticas centradas en la evolución genómica de la enfermedad ${ }^{8}$.

Esto ha permitido evidenciar mutaciones en diversos genes incluyendo el KIT (v-kit Hardy-Zuckerman 4 feline sarcoma viral oncogenehomolog) y el PDGFRA (Alpha-typeplatelet-derivedgrowth factor receptor) encontrados en el $80 \%$ y $10 \%$ de los pacientes, respectivamente. De manera análoga a otras neoplasias, estas alteraciones moleculares han modificado radicalmente el diagnóstico, tratamiento y el pronóstico de la enfermedad, permitiendo subseleccionar algunos de los manejos. La expresión cualitativa del KIT (CD117) determinada por inmunohistoquímica representa el estándar de oro para el diagnóstico en el contexto de una histología típica'; no obstante, hoy y en el futuro, será necesario conocer las variantes genómicas de la enfermedad.

El imatinib se considera el medicamento de elección para los pacientes con enfermedad irresecable o metastásica. A partir del 2001, se reportó el primer caso tratado con este medicamento, en el que se documentó una reducción significativa del volumen tumoral y una supervivencia libre de progresión (SLP) mayor a un año ${ }^{8}$. En nuestra población, Vargas y colaboradores encontraron que el imatinib proporciona una tasa esperada de respuesta global del $50 \%$, beneficio clínico del $88 \%$ y una SG que superó los 40 meses ${ }^{5}$. Eventualmente, la mayoría de los pacientes desarrollan resistencia al tratamiento de primera línea, por lo que se han incluido otras moléculas como el sunitinib, sorafenib y el regorafenib?.

Los genes KRAS (V-Ki-ras2 Kirstenrat sarcoma viral oncogenehomolog) y BRAF (serine/threonineproteinkinase B-Raf) juegan un papel fundamental en la carcinogénesis de diversos tumores, sin embargo, su significado en los pacientes con GIST aún es incierto ${ }^{10}$. Recientemente, Miranda y colaboradores encontraron una coactivación concomitante del KIT y del PDGFRA con el KRAS (5\%) y el BRAF (2\%), demostrando in vitro que el imatinib fue capaz de modificar la señalización a través del primero de estos genes, pero no los cambios ocasionados por los efectores del RAS/RAF ${ }^{11}$.

A continuación, se expone el caso de un paciente con diagnóstico de GIST de riesgo intermedio tratado con imatinib, sunitinib y sorafenib, en quien se encontró un genotipo complejo que permitió optimizar el tratamiento mejorando la SLP durante la tercera línea de intervención. Además, se exploran nuevos mecanismos sobre la configuración genética de la enfermedad y los patrones moleculares que explican la resistencia.

\section{Presentación del caso}

Se trata de un hombre de 35 años, sin antecedentes relevantes ni sintomatología previa, que presentó en julio del 2007 dolor abdominal severo de patrón visceral. Siguiendo la impresión diagnóstica de un abdomen agudo, fue llevado a una laparotomía exploratoria, donde se encontró una lesión tumoral en el yeyuno proximal de $110 \times 80 \times 40 \mathrm{~mm}$, sin otros hallazgos. El procedimiento finalizó sin complicaciones, logrando una resección total de la masa (R0) con anastomosis término-terminal.

El espécimen diagnóstico proveniente del primer procedimiento fue fijado en formalina tamponada y embebido en parafina, de la que se obtuvieron cortes de $5 \mu \mathrm{m}$ para realizar inmunohistoquímica, que incluyó los anticuerpos CD117, CD34, S100 y desmina (las fuentes de los anticuerpos, diluciones y protocolos de tinción se pueden obtener bajo solicitud al contacto). La patología evidenció un GIST de patrón fusocelular, 
con 6 mitosis en 40 campos, bordes libres de tumor y expresión positiva para CD117 y CD34, y negativa para S100 y enolasa. La enfermedad se clasificó como de alto riesgo.

El paciente entró en seguimiento clínico e imaginológico sin recibir tratamiento adyuvante. En diciembre del 2008, se documentó recaída al encontrar múltiples lesiones en el parénquima hepático. Inicialmente se consideraron como potencialmente resecables previa respuesta a imatinib, que comenzó con dosis de 400 mg/día a partir de febrero del 2009. El control imaginológico mostró progresión de la enfermedad, por lo que se duplicó la dosis del inhibidor en primera línea sin lograr beneficio clínico.

Se intentó la metastasectomía en julio de 2009, siendo fútil por encontrarse compromiso tumoral a nivel subdiafragmático. En agosto del mismo año, se inició sunitinib $50 \mathrm{mg} / \mathrm{d}$ áa (cuatro semanas de tratamiento continuo con suspensión en las dos siguientes), hallando
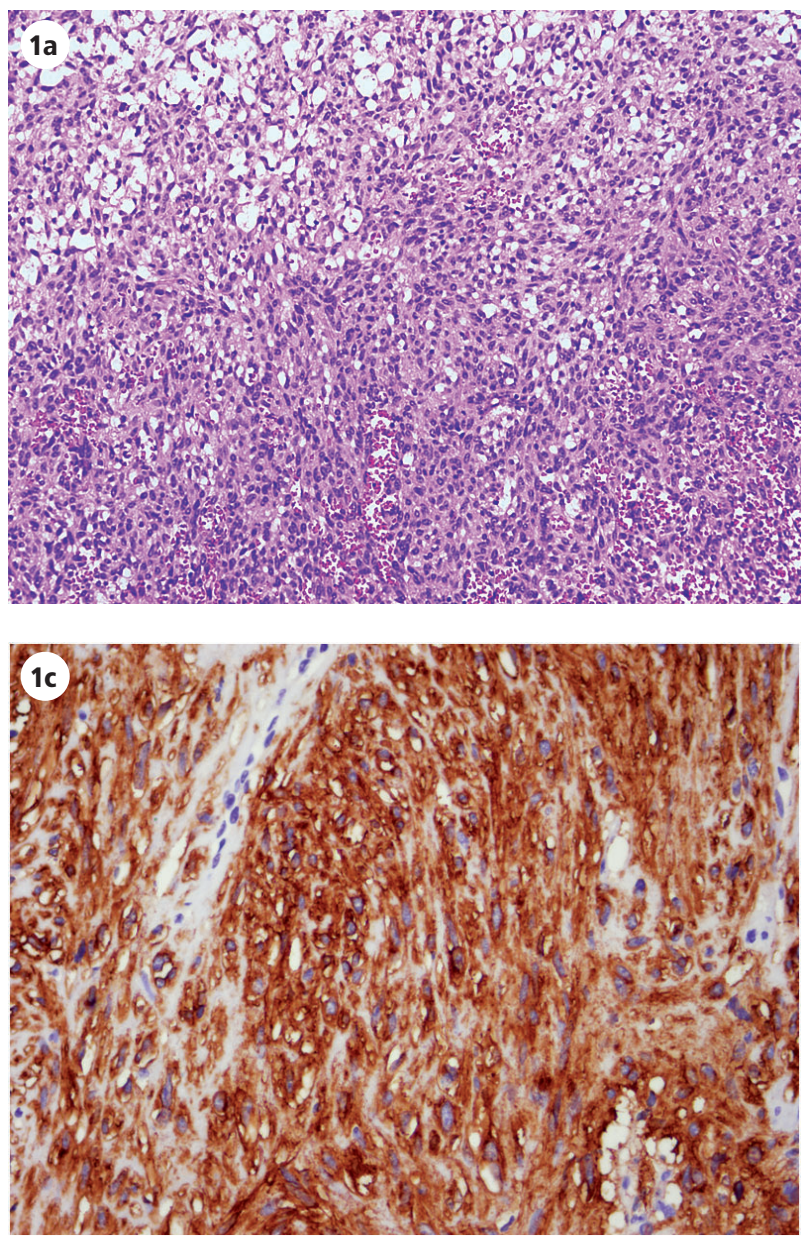

después de dos ciclos un mayor compromiso visceral, interpretado como progresión de la enfermedad (la evaluación no incluyó los criterios de Choi).

El comportamiento inusual de la enfermedad indicó una revisión del estudio histopatológico, en el que se encontró reactividad para CD117 en el 100\% de la población estudiada; por el contrario, no se documentó expresión para el CD34, actina muscular específica, desmina y S100. El índice de proliferación cuantificado por el Ki67 fue del 2\% y el conteo mitótico fue de 3 por 50 campos de alto poder (40x) (figura 1). A partir de estos hallazgos, el tumor se reclasificó como un GIST de riesgo intermedio, según la clasificación del NIH y AFIP12-14 (figura 1).

Ante la progresión, se llevó a cabo una tomografía por emisión de positrones (PET/TAC) basal que mostró el estado de la enfermedad (figura 2a) con compromiso hepático multifocal. Se inició sorafenib 400 mg cada 12 horas a partir de noviembre del 2009, hallando después
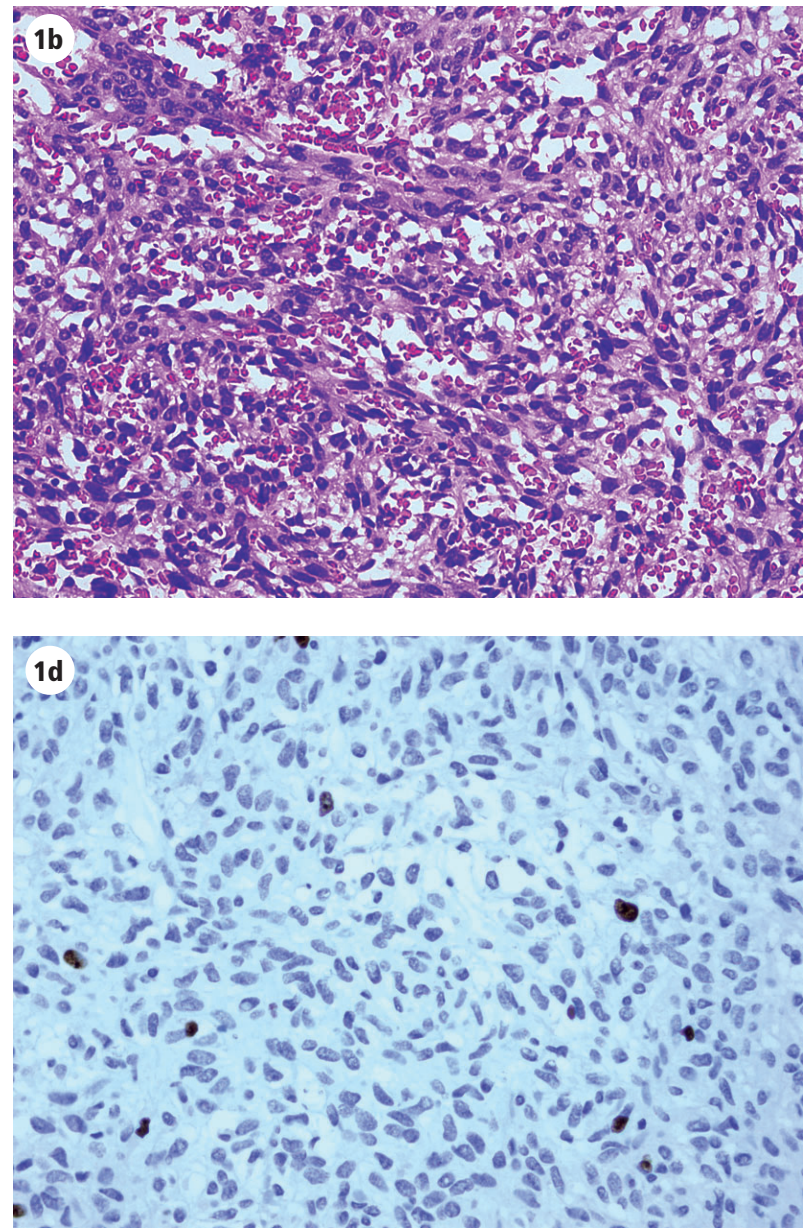

Figura 1. Hallazgos al examen histopatológico. a) Morfología de patrón fusocelular compatible con neoplasia del estroma gastrointestinal (20x). b) Histología con aumento de 40x. c) Reactividad de las células tumorales para CD117 en el 100\% de la población estudiada. d) Índice de proliferación Ki67\% (MIB-1) cuantificado en un 2\% y conteo mitótico de 3 por 50 campos de alto poder (40x). 

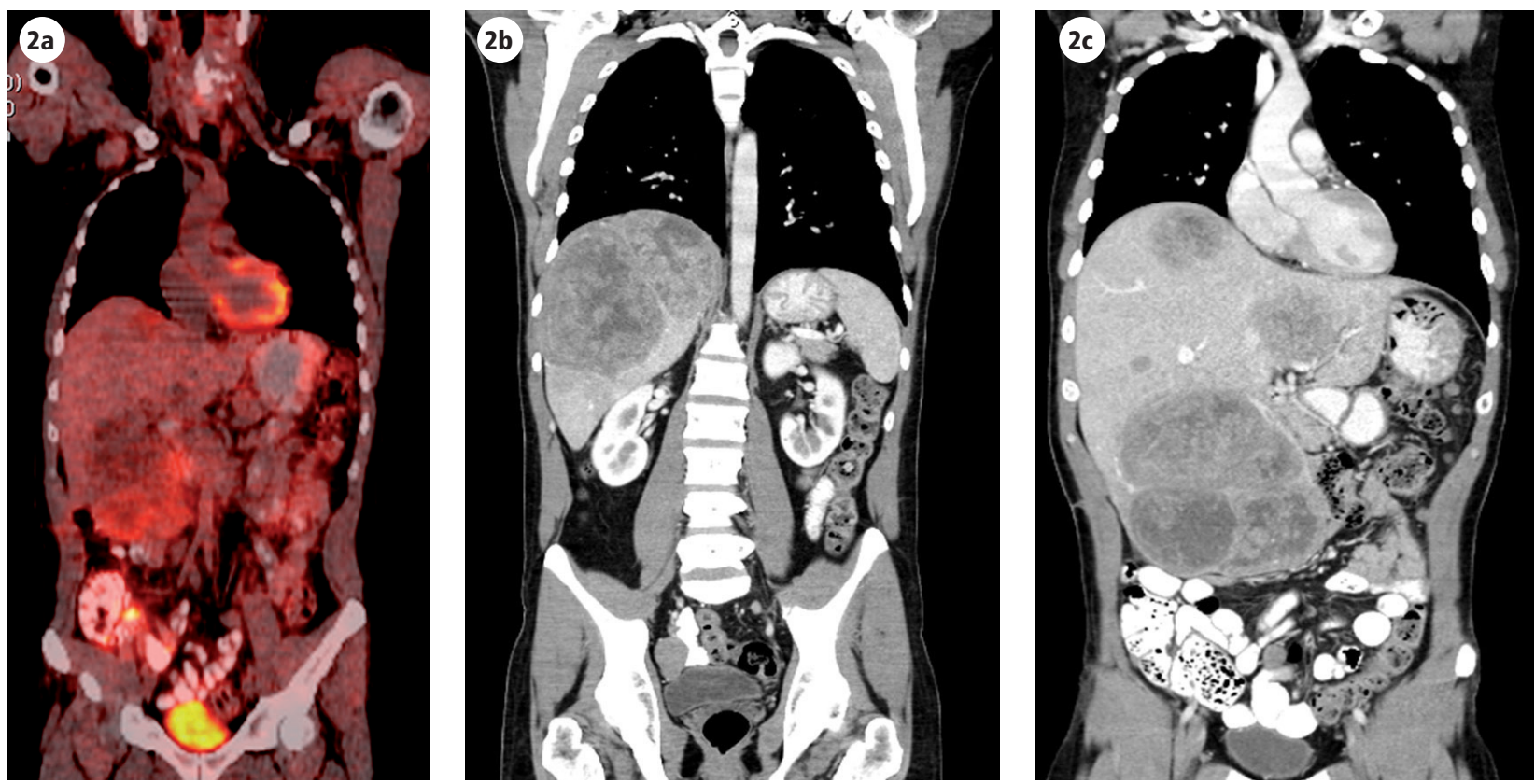

Figura 2. Imaginología del paciente. a) Estado basal de la enfermedad usando tomografía por emisión de positrones (previo inicio del sorafenib). b) Respuesta parcial máxima observada por criterios de Choi. c) Imagen al momento de la progresión tumoral después de 20 meses de tratamiento con sorafenib.

de dos ciclos una respuesta parcial con toxicidad aceptable dada por diarrea y dolor abdominal leve. Para explorar posibles mecanismos genéticos que explicaran el comportamiento de la patología, se extrajo ADN del tejido embebido en parafina, el cual se amplificó utilizando primers específicos para los exones 9 y 11 del KIT, mediante la técnica de reacción en cadena de la polimerasa (PCR).

En enero del 2010, se documentó e informó la presencia de una mutación en el exón 9 del gen KIT correspondiente a una duplicación de 3 pares de bases que involucraba los codones 502-503 (figura 3a). Durante el seguimiento, se mantuvo tanto la respuesta imaginológica como la clínica, y, en octubre del 2010, se hicieron estudios genéticos adicionales, incluyendo la secuenciación de los codones 2 y 3 del exón 15 del gen BRAF en el mismo tejido tumoral, encontrando positividad para la mutación V600E acompañada de una pérdida en la expresión de p16 determinada por inmunohistoquímica (figura 3b).

Para ese momento, se documentó una respuesta parcial máxima del 36\% siguiendo los criterios de Choi (figura 2b); el paciente recibió el sorafenib durante 20 meses, hasta julio del 2011, cuando se evidenció progresión de la enfermedad a nivel peritoneal con una moderada cantidad de líquido libre en cavidad, junto a la presencia de masas necróticas en el hemiabdomen derecho de $17 \mathrm{~mm}$, flancos derecho $(30 \mathrm{~mm}) \mathrm{e}$ izquierdo (16 mm), y en la pelvis (45 mm) (figura 2c), y unas lesiones hepáticas voluminosas de hasta $16 \mathrm{~cm}$ que reemplazaban el $80 \%$ del parénquima. Ante este hallazgo, se suspendió el sorafenib y se consideró una reinducción con sunitinib usando una dosis de $37,5 \mathrm{mg} /$ día y siguiendo la pauta 2-1-2-1 (administración durante dos semanas con una semana de suspensión, dos veces por ciclo). A la fecha, el paciente continúa vivo, sin deterioro en su calidad de vida ni en su funcionalidad.

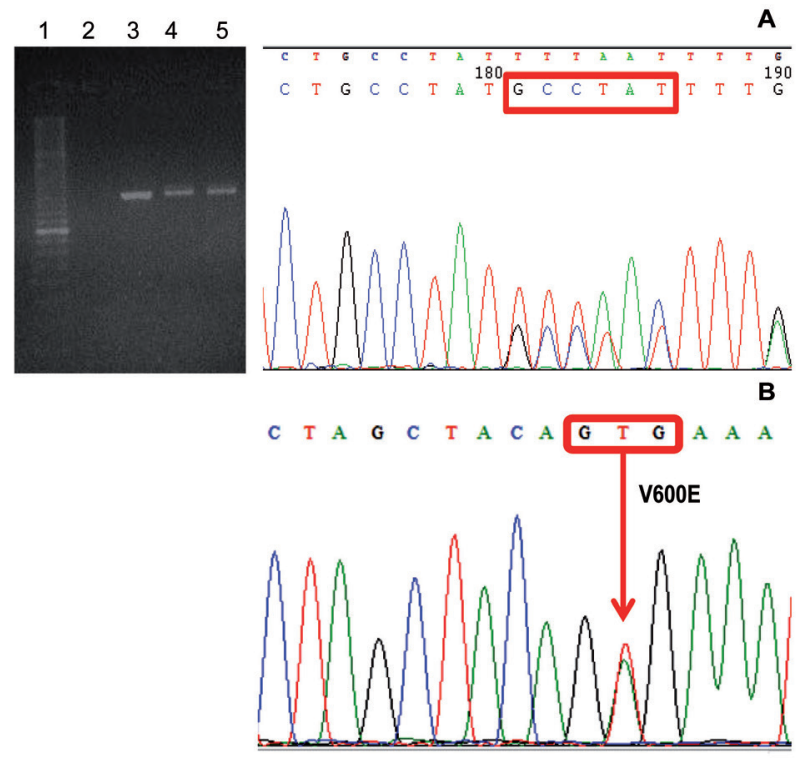

Figura 3. a) Corrido de electroforesis de los exones 9 del cKIT y cromatograma de la secuencia del mismo segmento que muestra una duplicación heterocigota de los codones 502-503. b) Cromatograma de la secuencia del exón 15 del BRAF que demuestra la mutación V600E. 


\section{Discusión}

EI GIST representa un tipo infrecuente de tumor de origen mesenquimal que afecta a hombres y mujeres en proporción similar, con una mediana para la edad del diagnóstico que oscila entre los 55 y 65 años. Con frecuencia, hay ausencia de síntomas, por lo que a menudo se manifiesta como un hallazgo incidental endoscópico o radiológico ${ }^{7}$. La clínica depende del tamaño y de la agresividad de la lesión, pudiendo presentarse dolor abdominal acompañado o no de una masa palpable, sangrado digestivo agudo o intercurrente, y obstrucción intestinal ${ }^{12}$. Entre la población colombiana, el síntoma más común fue el dolor abdominal, en el $64 \%$ de los casos, evento que se asoció con la detección de una masa en el sitio afecto ${ }^{6}$. En el presente caso, el cuadro clínico fue consistente con los signos descritos.

Al menos entre el $50 \%$ y $60 \%$ de los GIST se presentan como lesiones primarias al momento del diagnóstico ${ }^{14}$, y la enfermedad metastásica, que es menos frecuente, se demuestra entre el $15 \%$ y $50 \%$ de los pacientes $5,6,16$. De forma global, se acepta que las lesiones son de manejo quirúrgico, realizando resecciones segmentarias sin linfadenectomía, incluso por vía laparoscópica ${ }^{15}$. La inmunohistoquímica del GIST suele ser positiva para el cKIT (90\%-100\%), CD34 (70\%), actina de músculo liso (20\%-30\%), S100 (5\%) $y$, ocasionalmente, para desmina y queratina $(2 \%)^{14,17}$. En nuestro caso, la presentación clínica inicial siguió el curso usual, y la morfología y marcadores fueron compatibles con el diagnóstico; llama la atención la diferencia entre la evaluación inicial y la revisión, ya que se encontraron estados divergentes para el CD34 y el número de mitosis.

Durante los últimos 20 años, se han producido grandes avances en el entendimiento biológico del GIST, comportamiento que ha permitido caracterizar su fenotipo y diversas alteraciones moleculares que han sido valiosas para direccionar el uso de los inhibidores de tirosina-quinasa ${ }^{18}$. A partir del descubrimiento de la activación constitutiva del receptor de membrana producto del gen KIT, se describió el mecanismo esencial que soporta la oncogénesis de la enfermedad; la inhibición de su señal con el uso del imatinib y sunitinib surgió como alternativa primaria para los pacientes que no son candidatos a cirugía. A pesar de que la mayoría de los pacientes con mutaciones en el KIT alcanzan el beneficio clínico, algunos pueden presentar resistencia primaria o adquirida; esta última entre 12 y 36 meses después del inicio de la primera y segunda línea, respectivamente ${ }^{19}$.

Las mutaciones en el KIT y, en menor proporción, en el PDGFRA se consideran efectores primarios de la enfermedad, por lo que su identificación resulta importante antes de iniciar el inhibidor de tirosina-quinasa de primera línea. Fisiológicamente, la activación de un receptor proteico multimérico (como es el caso de KIT o PDGFRA) ocurre cuando se une el ligando, hecho que desencadena la dimerización del receptor, seguida de la autofosforilación del dominio de tirosina-quinasa intracelular y, por último, la activación final de múltiples sustratos incluidos en vías de señalización como PI3K/AKT, RAS, MAP y JAK/STAT.

Clínicamente, la activación de estas cascadas resulta en la promoción del ciclo celular, en la proliferación e inhibición de la apoptosis ${ }^{12,14}$. Sin embargo, existen mutaciones secundarias que se desarrollan en clonos seleccionados que aparecen durante o después del tratamiento, ocasionando resistencia. Este tipo de alteraciones afectan el dominio regulador o el enclave enzimático del receptor KIT o PDGFRA, lo que genera una estimulación constitutiva ${ }^{18,20}$.

Previamente, se encontró que las dos mutaciones descritas en el GIST son mutuamente excluyentes, incluso se describió un pequeño segmento de la enfermedad denominado "silvestre", que no presenta alteraciones en el KIT o en el PDGFRA ${ }^{21}$. Algunas series recientes han descrito la presencia de la mutación V600E del gen BRAF hasta en $7 \%$ de los pacientes con GIST, especialmente en los sujetos con un genotipo silvestre; este hallazgo no tiene una implicación biológica o pronóstica clara ${ }^{22}$

El BRAF es un miembro de la familia serina-treoninaproteína-quinasas, que funciona como efector de las proteínas $\mathrm{G}$ denominadas RAS, que están involucradas en la señalización vía MAPK/ERK, encargada de la transducción de señales extracelulares (iniciadas por los receptores KIT o PDGFRA) a la maquinaria reguladora de la transcripción (figura 4). Las proteínas RAF fosforilan a las proteína-quinasas selectivas MEK1 y MEK2 (MAPKKs), que a su vez activan a ERK1 y ERK2 (MAPKs), encargados de influir sobre los factores ETS11, c-JUN y c-MYC ${ }^{23}$. 


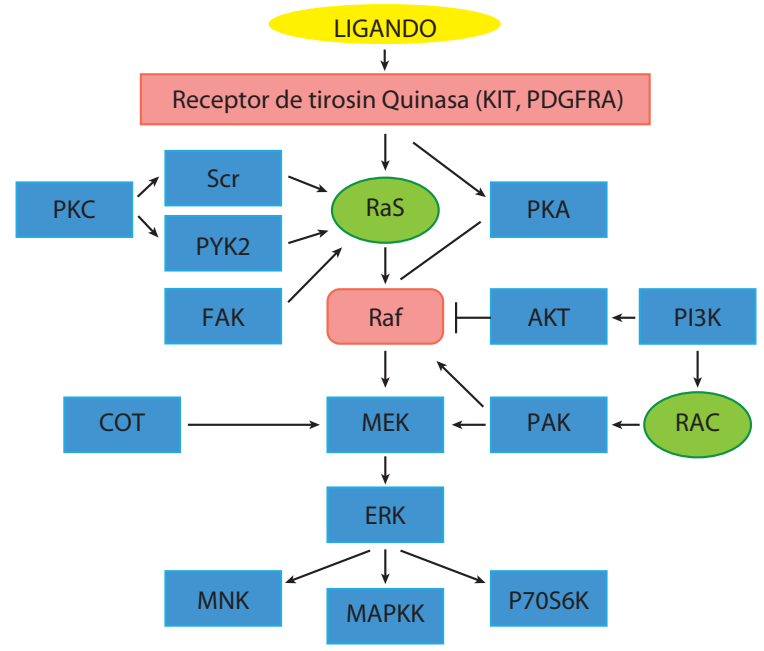

Figura 4. Vía de señalización del KIT/BRAF/MAP quinasas.

El melanoma representa el ejemplo clásico en el cual las mutaciones del BRAF tienen una actividad biológica esencial hasta en el $60 \%$ de los casos. Entre otros, el mismo gen está implicado en la patogénesis de neoplasias con estirpe epitelial, como el carcinoma papilar de tiroides, el cáncer colorrectal y la leucemia de células peludas ${ }^{24-26}$. La embriogénesis de las lesiones derivadas de los melanocitos y del mienterio comparten la expresión alterada del KIT, del BRAF y del EGFR22,27.

Hasta hace poco, no se habían detectado mutaciones en el KRAS en pacientes con GIST que presentaban alteraciones en el KIT; Antonescu y colaboradores identificaron mutaciones en el codón 12 (G12D: GGT $\rightarrow \mathrm{GaT}$ ), en el 13 (G13D: GGC $\rightarrow \mathrm{GaC}$ ), y una variación concomitante (G12A/G13D: GGT $\rightarrow$ GcT y GGC $\rightarrow \mathrm{GaC}$ ) en el KRAS en tres pacientes sin exposición previa al imatinib $(5 \%)^{28}$. Por otra parte, la mutación más frecuente del BRAF se localiza en el exón 15 (V600E), sin que se haya determinado hasta el momento ninguna alteración en el exón 11 del mismo gen o en el NRAS22. En nuestro caso, se demostró un genotipo complejo que incluyó la mutación del exón 9 del KIT y la presentación secundaria de la V600E en el BRAF, hallazgo que pudo condicionar un mecanismo dinámico de resistencia contra el imatinib.

Cerca del $50 \%$ de los pacientes que desarrollan resistencia al imatinib carecen de mecanismos identificables en los genes KIT o PDGFRA ${ }^{28}$; en este subgrupo, la génesis de mutaciones somáticas adquiridas podría explicar la disminución en la tasa de respuesta. Al menos un modelo permitió estudiar la hipótesis de la participación de las MAPK quinasas mediadas por el BRAF tras la inducción con los inhibidores de tirosinaquinasa, sin embargo, su comprobación requiere de la evaluación de otros estudios preclínicos in vivo ${ }^{28}$.

La detección de mutaciones del BRAF en sujetos con GIST conlleva un impacto trascendental en la clínica. En primer lugar, se identificó un número de pacientes silvestres para KIT y PDGFRA menos sensibles al imatinib que podrían beneficiarse de mecanismos farmacológicos alternativos, incluyendo medicamentos como el sorafenib, regorafenib y el vemurafenib. En segundo lugar, hace poco se encontró que la mutación V600E se asocia en el GIST a un aumento en la sensibilidad a la inhibición selectiva del MEK, lo que podría constituir un blanco terapéutico potencial para los inhibidores específicos ${ }^{29}$.

Los primeros reportes de las alteraciones en el BRAF en pacientes con GIST desconocieron el efecto de las mutaciones complejas sobre las intervenciones terapéuticas. Rossi y colaboradores evaluaron el papel de la señalización del KIT sobre ERK1/2, demostrando que estas proteínas pueden estar activadas o no tras la modificación del KIT, evento que permite plantear al BRAF como un efector alternativo en los casos en los que se encuentra un bloqueo ineficaz sobre el KIT ${ }^{30}$. Este supuesto puede justificarse a partir del análisis de los estudios que han utilizado efectivamente inhibidores directos del BRAF en otras patologías ${ }^{31-33}$.

Se han explorado pocos medicamentos como alternativas de tercera línea para el manejo del GIST irresecable o metastásico. El nilotinib es un inhibidor de tirosina-quinasa de segunda generación que actúa por inhibición competitiva del ATP para facilitar la unión del producto del gen de fusión BCR-ABL en leucemia mieloide crónica. Un estudio fase II en pacientes con GIST no demostró resultados positivos ${ }^{34}$. El sorafenib inhibe el CKIT, VEGFR, PDGFR-ß y varias quinasas de la familia RAF. In vitro se demostró actividad para inhibir células de GIST resistentes a imatinib y sunitinb ${ }^{35}$, lo que conllevó a evaluar su eficacia en pacientes politratados, hallando una respuesta parcial y enfermedad estable en el $14 \%$ y $64 \%$, respectivamente ${ }^{32}$. Una actualización de este estudio mostró una mediana de SLP de 5.2 meses (IC 95\% 3.4-7.4 meses) y una SG de 11.6 meses (IC $95 \% 8.8-14.3)^{33}$, desenlaces respaldados por otra serie de corte transversal ${ }^{36}$. 
El caso aquí reportado tuvo un efecto terapéutico extrapolable a lo encontrado en modelos murinos que tienen resistencia primaria al imatinib mediada por la mutación V600E del BRAF. El hallazgo más significativo fue la extensa SLP con el uso del sorafenib, que superó los resultados descritos por Kindler y colaboradores, que no discriminaron la presencia o ausencia de alteraciones en el BRAF ${ }^{33}$. La respuesta clínica obtenida sugiere una dominancia del BRAF sobre el KIT para activar las vías MAPK/ERK, lo que sugiere utilizar en líneas posteriores un inhibidor directo (MEK162, SU11248 y PD0325901).

En el GIST, el paradigma de que una sola alteración genética explica el mecanismo biológico de la enfermedad es en la actualidad un punto controvertido ${ }^{18,28}$. Además de las alteraciones en el BRAF aisladas o

Tabla 1. Medicamentos en investigación actual para pacientes con GIST que progresan a imatinib o sunitinib

\begin{tabular}{|c|l|l|c|}
\hline Fase & \multicolumn{1}{|c|}{ Molécula } & \multicolumn{1}{|c|}{ Patrocinador } & $\begin{array}{c}\text { Número de referencia } \\
\text { del estudio* }\end{array}$ \\
\hline$\|$ & Pazopanib & GSK & NCT01391611 \\
\hline$\|$ & AT13387 & Astex Pharmaceuticals & NCT01294202 \\
\hline$\|$ & AYU922 & Novartis & NCT01389583 \\
\hline$\|$ & STA-9090 (ganetespib) & $\begin{array}{l}\text { Synta Pharmaceuticals } \\
\text { Corp. }\end{array}$ & NCT01039519 \\
\hline$\|$ & Dovitinib & Novartis & NCT01440959 \\
\hline IIb & BKM120 & Novartis & NCT01468688 \\
\hline
\end{tabular}

${ }^{*}$ Clinicaltrials.gov (<http://www.clinicaltrials.gov>) conjuntas con el KIT y el PDGFRA ${ }^{37}$, se han descrito variaciones en PI3KCA y en PTEN ${ }^{19}$. Algunos estudios exploran opciones farmacológicas dirigidas contra estas vías y otras encargadas de caracterizar la variabilidad farmacocinética ligada a cambios metabólicos en enzimas transportadoras (tabla 1)

\section{Conclusión}

El comportamiento biológico del GIST del paciente reportado representa un ejemplo claro de resistencia primaria que alcanzó un efecto terapéutico positivo con el sorafenib. Este hecho respalda la exploración sistemática del genotipo, incluyendo en el futuro alteraciones en el BRAF, KRAS y PI3K. La evolución biológica de esta enfermedad nos permitió realizar una correlación de transferencia in vivo, personalizando el tratamiento para maximizar el beneficio. Es posible que, en el futuro, los pacientes con la mutación del exón 9 del KIT, con la variación D842V del exón 18 del PDGFRA y del BRAF, no se beneficien de recibir imatinib; por ahora, el grupo conocido como GIST silvestre se puede reclasificar en sujetos positivos para BRAF, KRAS o PI3K, hallazgos que podrían cambiar el perfil diagnóstico y el tratamiento.

\section{Referencias}

1. Rossi CR, Mocellin S, Mancarelli R, Foletto M, Pilati P, Nitti D, et al. Gastrointestinal stromal tumours: from a surgical to a molecular approach. Int J Cancer. 2003;107(2):171-6.

2. Duffaud F, Blay JY. Gastrointestinal stromal tumors: biology and treatment. Oncology. 2003;65(3):187-97.

3. Thuneberg L. Interstitial cells of Cajal: intestinal pacemaker cells? Adv Anat Embryo Cell Biol. 1982;71:1-130.

4. Chan JK. Mesenchymal tumours of the gastrointestinal tract: a paradise for acronyms (STUMP, GIST, GANT, and now GIPACT). Implications of c-kit in genesis, and yet another of many emerging roles of the interstitial cell of Cajal in the pathogenesis of gastrointestinal disease? Adv Anat Pathol. 1999;6(1):19-40.

5. Vargas C, Cardona AF, Carranza H, Otero JM, Reveiz L, Ospina $E$, et al. Tumores del estroma gastrointestinal (GIST): experiencia en dos instituciones hospitalarias de Bogotá D.C., Colombia (estudio del ONCOLGroup). Rev Col Gastroenterol. 2008;23(3):213-23

6. Oliveros R, Quintero AP, Sánchez R, Mesa JA. Tumores estromales gastrointestinales (GIST) en el Instituto Nacional de Cancerología (INC) 2000-2008. Rev Col Cancerol. 2011;15(4):1-34.

7. Nickl NJ. Gastrointestinal stromal tumors: new progress, new questions. Curr Opin Gastroenterol. 2004;20(5):482-7.

8. Joensuu H, Roberts P, Sarlomo-Rikala M, Andersson LC, Tervahartiala $P$, Tuveson D, et al. Effect of the tyrosine kinase inhibitor STI571 in a patient with a metastatic gastrointestinal stromal tumor. N Engl J Med. 2001;344(14):1052-6.

9. Joensuu $H$. Second line therapies for the treatment of gastrointestinal stromal tumor. Curr Opin Oncol. 2007;19(4):3538.

10. Rajagopalan H, Bardelli A, Lengauer $C$, Kinzler KW, Vogelstein $B$, Velculescu VE. Tumorigenesis: RAF/RAS oncogenes and mismatch-repair status. Nature. 2002;418(6901):934.

11. Miranda C, Nucifora M, Molinari M, Conca E, Anania MC, Bordoni $A$, et al. KRAS and BRAF mutations predict primary resistance to imatinib in gastrointestinal stromal tumors. Clin Cancer Res. 2012;18(6):1769-76.

12. Miettinen M, Lasota J. Gastrointestinal stromal tumors: pathology and prognosis at different sites. Semin Diagn Pathol. 2006;23(2):70-83

13. Liegl-Atzwanger B, Fletcher JA, Fletcher CD. Gastrointestinal stromal tumors. Virchows Arch. 2010;456(2):111-27.

14. Fletcher CD, Berman JJ, Corless C, Gorstein F, Lasota J, Longley 
BJ, et al. Diagnosis of gastrointestinal stromal tumors: a consensus approach. Hum Pathol. 2002;33(5):459-65.

15. Fernández JA, Sánchez Canovas M, Parrilla P. Controversias en el tratamiento de los tumores del estroma gastrointestinal (GIST) primarios. Cir Esp. 2010;88:69-80.

16. Scarpa M, Bertin M, Ruffolo C, Polese L, D'Amico DF, Angriman I. A systematic review on the clinical diagnosis of gastrointestinal stromal tumors. J Surg Oncol. 2008;98(5):384-92.

17. van de Rijn M, Hendrickson MR, Rouse RV. CD34 expression by gastrointestinal tract stromal tumors. Human Pathol. 1994:25(8):766-71

18. Downs-Kelly E, Rubi BP. Gastrointestinal stromal tumors: molecular mechanisms and targeted therapies. Patholog Res Int. 2011;4:1-7.

19. Daniels M, Lurkin I, Pauli R, Erbstösser E, Hildebrandt U, Hellwig $\mathrm{K}$, et al. Spectrum of KIT/PDGFRA/BRAF mutations and Phosphatidylinositol-3-Kinase pathway gene alterations in gastrointestinal stromal tumors (GIST). Cancer Lett. 2011;312(1):43-54.

20. Longley BJ, Reguera MJ, Ma Y. Classes of C-KIT activating mutations: proposed mechanisms of action and implications for disease classification and therapy. Leuk Res. 2001;25(7):571-6.

21. Medeiros F, Corless CL, Duensing A, Hornick JL, Oliveira AM, Heinrich MC, et al. KIT-negative gastrointestinal stromal tumors. Am J Surg Pathol. 2004;28(7):889-94.

22. Agaram NP, Wong GC, Guo T, Maki RG, Singer S, Dematteo $R P$, et al. Novel V600E BRAF mutations in imatinib-naive and imatinib-resistant gastrointestinal stromal tumors. Genes Chromosomes Cancer. 2008;47(10):853-9.

23. Davies H, Bignell GR, Cox C, Stephens P, Edkins S, Clegg S, et al. Mutations of the BRAF gene in human cancer. Nature. 2002;417(6892):949-54.

24. Brose MS, Volpe P, Feldman M, Kumar M, Rishi I, Gerrero R, et al. BRAF and RAS mutations in human lung cancer and melanoma. Cancer Res. 2002;62(23):6997-7000.

25. Tiacci E, Trifonov V, Schiavoni G, Holmes A, Kern W, Martelli $M P$, et al. BRAF mutations in hairy-cell leukemia. N Engl J Med. 2011;364(24):2305-15.

26. Michaloglou C, Vredeveld LC, Mooi WJ, Peeper DS. BRAF (E600) in benign and malignant human tumours. Oncogene. 2008;27(7):877-95

27. Curtin JA, Fridlyand J, Kageshita T, Patel HN, Busam KJ, Kutzner $\mathrm{H}$, et al. Distinct sets of genetic alterations in melanoma. N Engl J Med. 2005;353(20):2135-47.
28. Antonescu CR, Besmer P, Guo T, Arkun K, Hom G, Koryotows$\mathrm{ki} B$, et al. Acquired resistance to imatinib in gastrointestinal stromal tumor occurs through secondary gene mutation. Clin Cancer Res. 2005;11(11):4182-90.

29. Solit DB, Garraway LA, Pratilas CA, Sawai A, Getz G, Basso A, et al. BRAF mutation predicts sensitivity to MEK inhibition. Nature. 2006;439(7074):358-62.

30. Rossi F, Ehlers I, Agosti V, Socci ND, Viale A, Sommer G, et al. Oncogenic Kit signaling and therapeutic intervention in a mouse model of gastrointestinal stromal tumor. Proc Natl Acad Sci USA. 2006;103(34):12843-8.

31. Chapman PB, Hauschild A, Robert $C$, Haanen JB, Ascierto P, Larkin J, et al. Improved survival with vemurafenib in melanoma with BRAF V600E mutation. N Engl J Med. 2011;364(26):2507-16.

32. Nimeiri HS, Maki RG, Kasza K, D'Adamo D, Chow W, et al. Activity of sorafenib (SOR) in patients (pts) with imatinib (IM) and sunitinib (SU)-resistant (RES) gastrointestinal tumors (GIST): A phase II trial of the University of Chicago Phase II Consortium. J Clin Oncol. 2008;26:abstr 10502.

33. Kindler HL, Campbell NP, Wroblewski K, Maki RG, D'Adamo DR, Chow WA, et al. Sorafenib in patients with imatinib and sunitinib resistant gastrointestinal stromal tumors (GIST): Final results of a University of Chicago Phase II Consortium trial. J Clin Oncol. 2011;29:abstr 10009.

34. Cauchi C, Somaiah N, Engstrom P, Litwin S, López M, Lee L, et al. Evaluation of nilotinib (N) in advanced GIST previously treated with imatinibmesylate (IM) and sunitinib (S). J Clin Oncol. 2010;28:15s, abstr 10090.

35. Guo T, Agaram NP, Wong GC, Hom G, D'Adamo D, Maki RG, et al. Sorafenib inhibits the imatinib-resistant KITT670I gatekeeper mutation in gastrointestinal stromal tumor. Clin Cancer Res. 2007;13(16):4874-81

36. Italiano A, Cioffi A, Maki RG, Schöffski P, Rutkowski P, Le Cesne $A$, et al. Patterns of care, prognosis, and survival of patients with metastatic gastrointestinal stromal tumors (GIST) refractory to first-line imatinib and second-line sunitinib. J Clin Oncol. 2011;29:abstr 10044.

37. Agaram NP, Laquaglia MP, Ustun B, Guo T, Wong GC, Socci ND, et al. Molecular characterization of pediatric gastrointestinal stromal tumors. Clin Cancer Res. 2008;14(10):3204-15.

38. Pierotti MA, Tamborini E, Negri T, Pricl S, Pilotti S. Targeted therapy in GIST: in silico modeling for prediction of resistance. Nat Rev Clin Oncol. 2011;8(3):161-70. 